\title{
Notas sobre algunas gramíneas (Poaceae: Poeae) de la flora ibérica
}

Carlos Romero-Zarco', Llorenç Sáez ${ }^{2,3}$ \& Ana Teresa Romero-García ${ }^{4}$

${ }^{1}$ Departamento de Biología Vegetal y Ecología, Universidad de Sevilla, España.

${ }^{2}$ Systematic and Evolution of Vascular Plants (UAB), Associated unit to CSIC, Dept. BABVE, Faculty of Biosciences, Autonomous University of Barcelona. ES-08193, Bellaterra, Barcelona, Spain.

${ }^{3}$ Societat d'Història Natural de les Balears (SHNB), Margarida Xirgu, 16, ES-07003 Palma de Mallorca, Balearic Islands, Spain. ${ }^{4}$ Departamento de Botánica, Facultad de Ciencias, Universidad de Granada, España.

\section{Correspondencia}

e-mail: zarco@us.es

Recibido: 7 febrero 2021

Aceptado: 24 febrero 2021

Publicado on-line: 5 marzo 2021

Editado por: Marta Recio Criado

\section{Resumen}

Se aportan datos corológicos y nomenclaturales de algunas especies de gramíneas de la tribu Poeae (Poaceae) pertenecientes a los géneros Agrostis, Aira, Neoschischkinia y Holcus.

Palabras clave: Gramíneas, Poaceae, nomenclatura, distribución, Península Ibérica.

\begin{abstract}
Notes on some grasses (Poaceae: Poeae) from de Iberian flora

Chorological and nomenclatural data are provided for some species of grasses of the tribe Poeae (Poaceae) of the genera Agrostis, Aira, Neoschischkinia and Holcus.
\end{abstract}

Key words: Grasses, Poaceae, nomenclature, distribution, Iberian Peninsula.
Reunimos aquí algunas observaciones corológicas o nomenclaturales sobre gramíneas ibéricas que, en los géneros Aira y Holcus corrigen algo de lo ya publicado en Flora iberica XIX(I), mientras que en los géneros Agrostis y Neoschischkinia justifican información que actualmente está en proceso de edición para el volumen XIX(II) de la misma obra.

Agrostis curtisii Kerguélen in Bull. Soc. Bot. France 123(5-6): 318 (1976)

ESPAÑA. Córdoba. Entre Fuente Obejuna y La Granjuela, suelo aluvial, 30-V-1984, J. Arroyo et al. (SEV 204901).

Novedad para la provincia de Córdoba y para Sierra Morena. Especie de afinidad atlántica y silicícola que en Andalucía solo es relativamente frecuente en la provincia de Cádiz (Romero-Zarco, 1987). También se conoce de Ayamonte, en el sur de Huelva (Paunero, 1948) y del occidente de Málaga (Aparicio, 1987; Romero-García, 2011). Una cita de Sierra Nevada, en la provincia de Granada (Paunero, 1948), fue desestimada por Romero-García et al. (1988) quienes, tras infructuosa búsqueda, la consideraron como un error de etiquetado.

Agrostis $\times$ aquitanica Romero Zarco \& Romero García, nom. nov.

A. castellana $\times A$. stolonifera

A. $\times$ hackelii Fouill. in Bull. Soc. Bot. France 79: 801 (1933), nom. illeg., non E.R. Fr. (1905), syn. subst. Indicación locotípica: Tonnay-Charente [Charente Marítimo, Francia]
Lectotipo: P00572975, ejemplar de la derecha (anotado en el pliego por R. Portal).

Los detalles de la descripción de este híbrido se comentan en la nota siguiente. El epíteto aquitanica alude a la región del oeste de Francia de donde procede el material original.

Agrostis $\times$ fouilladei P. Fourn., Quatre FI. France: 49 (1934)

A. capillaris $\times A$. castellana

A. $\times$ fouilladeana Lambinon \& Verloov in Bull. Soc. Échange PI. Vasc. Eur. Occid. Bassin Médit. 29: 105 [-109] (2004), nom. illeg.

Fournier (1934) publica su nombre para el híbrido "A. castellana $\times$ vulgaris", siendo A. vulgaris With. sinónimo de $A$. capillaris $L$. No hay descripción ni referencia directa, pero sí hay una clara referencia al mismo híbrido descrito un año antes, aunque no nombrado, por Fouillade (1933: 801-803). El hecho de que Fournier llamara a este híbrido Fouilladei, es una clara referencia indirecta (aunque sea algo críptica) a una descripción anterior y cumple los requisitos para ser considerado un nombre válido según el artículo 38.14 del código (Greuter \& Rankin Rodríguez, 2018). Fouillade (op. cit.) describe con todo lujo de detalles tres híbridos que comentamos seguidamente.

1) A. capillaris $\times$ A. stolonifera

En la página 799 de su artículo recoge la descripción de S.S. Murbeck para una planta escandinava que identifica con el híbrido "A. stolonifera $\times$ 
vulgaris" y seguidamente, propone el nombre $A$. murbeckii en honor a su descubridor.

2) A. castellana $\times A$. stolonifera

En la página 800 , bajo el epígrafe "A. alba $\times$ castellana", comenta largamente una planta descrita previamente por E. Hackel como "A. alba ad castellanam vergens", llegando a la conclusión de que se trata del híbrido entre esas dos especies. En la página siguiente (801) justifica su nombre: "Je crois juste de dédier cet hybride, sous le nom $d$ '× $A$. HACKELII, à la mémoire du botaniste qui a le premier étudié les intermédiaires entre $A$. alba et $A$. castellana" ( $A$. alba auct., non $L$. es sinónimo de $A$. stolonifera).

\section{3) A. capillaris $\times A$. castellana}

En la página 801 , bajo el epígrafe "A. castellana $\times$ vulgaris" comienza una larga discusión sobre plantas recogidas por él mismo en los alrededores de Tonnay-Charente (departamento Charente Marítimo, $W$ de Francia) y para las que no encuentra precedente en la literatura del género. Este tercer híbrido es minuciosamente descrito aunque no nombrado, pues siguiendo la metodología de su trabajo debería serle dedicado a él mismo. Posteriormente, Fournier, de forma muy lógica y adecuada, siguiendo el mismo razonamiento, le dedica el tercer híbrido a su descubridor y primer descriptor, A. Fouillade. Es preciso aclarar que la interpretación que se hace en Tropicos.org del nombre $A$. $\times$ fouilladei, como nombre de sustitución de $A$. $\times$ hackelii, es completamente errónea, son híbridos distintos que comparten solo un parental: A. castellana.

Por su parte, la aseveración de Lambinon \& Verloove (2004) de que $A$. $\times$ fouilladei es un nomem nudum, es simplista y no tiene en cuenta la intención implícita de Fournier de nombrar el híbrido descrito por Fouillade. En consecuencia, $A . \times$ fouilladeana Lambinon \& Verloove in Bull. Soc. Échange PI. Vasc. Eur. Occid. Bassin Médit. 29: 105 [-109] (2004), con la misma fórmula híbrida de $A$. × fouilladei, aunque se describe como híbrido nuevo con su propio tipo, resulta supérfluo.

Aira caryophyllea L., Sp. PI.: 66 (1753), sensu stricto ESPAÑA. Cuenca. Cañete, XK12, 1000 m.s.m., areniscas triásicas, 24-V-1974, A. González \& G. López nํㅜㄹ 1279 GF (MA 447886). Huesca. Bielsa, VVII, sin año de recolección, C. del Campo (MA 86681). Sallent, llano de Formigal, IX-1850, Vallier (MA 7564). San Juan de la Peña, 14-VI-1970, Fernández-Casas (MA 409140). Madrid. Braojos, in pascuis arenosis, 2-VI-1918, C. Vicioso (MA 7526). Somosierra, sin fecha, Cutanda (MA 7527); íbidem, 1400 m.s.m., 26VII-1933, Cuatrecasas (MA 183698). Valladolid. Cigales, 30T UM5430, 850 m.s.m., claros de encinar en paramera calcárea, 6-VI-2008, J.A. Lázaro Bello (MA 794255). Zamora. Cubo del Vino, 17-V-1981, X. Giráldez (SALA 30606).

Hay numerosas citas para estas cinco provincias, pero no se había comprobado hasta el momento cuáles de ellas correspondían a la especie sensu stricto y cuales a otras especies, como $A$. multiculmis
Dumort. o A. hercynica Romero Zarco \& al., con las que se confunde fácilmente. La especie tiene una amplia distribución en la Península, si bien aún no se ha confirmado su presencia en algunas provincias. Una de ellas es Almería, que se incluyó en la lista de Flora iberica 19(1) (Sáez et al., 2020c) en base a un único pliego (SEV 287133), presuntamente procedente de Sierra Nevada, cuya veracidad queda en entredicho tras una atenta lectura del artículo de Benedí \& Sáez (1996) sobre ciertas plantas con etiquetas falsificadas.

Aira hercynica Romero Zarco, M.Á. Ortiz \& L. Sáez in Syst. Bot. 45(1): 81 (2020)

ESPAÑA. Ávila. Tremedal, 30T TK7771, Tuberarietalia, 1640 m.s.m., 20-VII-1992, S. Sardinero (MAF 142014, sub A. caryophyllea subsp. caryophyllea). PORTUGAL. Beira Litoral. Figueira da Foz, Serra de Boa Viagem, mirador, peñascos costeros, 29T NE0748, 280 m.s.m., 25-V-1972, $P$. Montserrat (JACA 195272, sub A. caryophyllea subsp. multiculmis).

Estos dos pliegos no se revisaron a tiempo para incluir estas provincias en el volumen XIX(I) de Flora iberica. La localidad de Ávila establece un límite altitudinal muy por encima del conocido hasta la fecha, pasando de 1260 a 1640 m.s.m. La especie es endémica de la Península Ibérica y su distribución conocida, bastante amplia, puede consultarse en Sáez et al. (2020a). La lista de testimonios se publicó en Sáez et al. (2020b).

Aira multiculmis Dumort., Observ. Gramin. Belg.: 121 (1824)

ESPAÑA. Navarra. Marañón-Cabredo, 30T WN4520, 700 m.s.m., 23-VI-1985, l. Aizpuru et. al., no 2109.85 (MA 364210). Soria. Barahona, calizo, 1000 m.s.m., 16-V-1964, A. Segura Zubizarreta (MA 363745, ejemplar № 1). Borobia, pr. Mata La Mocha, 30T WM9224, 1100 m.s.m., 6-VI-1994, G. Mateo et. al. no 8950-GM (MA 546375, sub A. caryophyllea). Cueva de Águeda, 30T WN9424, 1300 m.s.m., 22-VI1988, D. Gómez et. al. (JACA 196288, sub $A$. caryophyllea). El Royo, pasto pobre, 1300-1400 m.s.m., 3-VII-1958, P. Montserrat \& N.Y. Sandwith (JACA 46258).

Al igual que en el caso de $A$. caryophyllea, hay citas previas en ambas provincias, pero no se confirmaron a tiempo para incluirlas en distribución de Flora iberica.

Neoschischkinia nebulosa (Boiss. \& Reut.) Tzvelev in Bot. Zhurn. (Moscow \& Leningrad) 53: 309 (1968) Agrostis nebulosa Boiss. \& Reut.

ESPAÑA. Sevilla. Constantina, desembocadura del arroyo de las Truchas, 13-VI-1996, $R$. Tamajón (COFC 25269, sub Agrostis stolonifera).

Novedad provincial. Especie endémica de la Península lbérica, donde se distribuye principalmente por la mitad oriental, en consonancia por su preferencia por los terrenos calcáreos. En Andalucía occidental se conocía solo del sur de Córdoba 
(Romero-Zarco, 1987), pero no de Sierra Morena, donde este tipo de materiales está muy localizado. Hay además una cita antigua para terrenos arenosos y yesíferos cercanos a La Carlota, en la Campiña cordobesa, que completa la distribución de la especie en la provincia (Willkomm, 1861).

Holcus durieui Steudel, Nomencl. Bot., ed. 2 1: 772 (1840)

H. gayanus Boiss., Voy. Bot. Espagne 2: 637 (1842)

ESPAÑA. Segovia. Cuéllar, coto de la Peguera, borde de la hoz, 30TVL0074, 830 m.s.m., 8-VII-1998, Soriano Martín (MA 755451, ํㅡㄹ, mezclada con Aira multiculmis).

Novedad provincial y única población conocida en la parte central de la meseta Norte. Especie endémica del centro y oeste de la Península lbérica, donde crece con preferencia en sustratos rocosos silíceos. Las poblaciones más próximas conocidas se encuentran en la vertiente madrileña del Sistema Central (Fernández González, 1982, 1988).

En la lista de especies del género Holcus de la obra citada de Steudel, figura "Durieui. Steud. Asturia [sic]" seguida de la siguiente referencia que lo valida: "H. tenuis. Gay. (An. des sc. nat. 1836)". Laínz (1959) ya advirtió que el nombre de Steudel es legítimo y prioritario sobre el más conocido de Boissier. En las bases de datos solo se recoge una referencia posterior de $H$. durieui [Steudel, Syn. PI. Glumac. 1(1): 15 (1853)], lo que explica que en las floras básicas haya seguido apareciendo esta especie como $H$. gayanus. No obstante, la opinión de Laínz fue recogida recientemente (Romero-Zarco, 2015) sin que haya sido suficiente para rehabilitar el binomio de Steudel en Flora iberica.

\section{Holcus durieui var. pauneroae Romero Zarco, var. nova}

H. gayanus var. biaristatus Paunero in Anales Inst. Bot. Cavanilles 13: 165 (1956), nom. nud.

A typo differt flosculo inferiore aristato, basi piloso. Holotypus: León. Sierra del Teleno, sin fecha, Bernis (MA 7257).

En las especies ibéricas de la sección Holcus (v.gr. $H$. annuus C.A. Mey., H. durieui Steudel, $H$. lanatus L., H. mollis L. y $H$. reuteri Boiss.) la flor apical suele ser masculina -en ocasiones estéril- y aristada, mientras que la basal es femenina y rara vez lleva arista. En $H$. durieui son relativamente frecuentes las plantas con espiguillas biaristadas lo que llevó a Paunero (1956) a describir en español la variedad que aquí le dedicamos. Por el contrario, en las especies de la sección Homalachne Benth. \& Hook. $(H$. caespitosus Boiss. y $H$. grandiflorus Boiss. \& Reut.) ambas flores son hermafroditas y aristadas.

\section{Agradecimientos}

Agradecemos la amabilidad del personal de los herbarios mencionados $y$, de forma especial, a los doctores Leopoldo Medina y Francisco Javier Salgueiro, conservadores de los herbarios del Jardín
Botánico de Madrid (MA) y de la Universidad de Sevilla (SEV) respectivamente, por su atenta gestión.

\section{Bibliografía}

Aparicio, A. (1987). Contribución a la flora de la provincia de Málaga. Acta Botanica Malacitana, 12, 223-228.

Benedí, C. \& Sáez, L. (1996). Propósitos y despropósitos de Reineck y sus prosélitos. Anales del Jardín Botánico de Madrid, 54, 570-574.

Fernández González, F. (1982). Notas florísticas sobre el valle del Paular (Madrid, España). II. Lazaroa, 4, 375-378.

Fernández González, F. (1988). Estudio florístico y fitosociológico del valle del Paular, Tesis doctoral. Facultad de Biología. Univ. Complutense de Madrid.

Fouillade, M. (1933). Sur les Agrostis alba, vulgaris, castellana et leurs hybrides. Bulletin de la Société Botanique de France, 79, 789-804.

Fournier, P. (1934). Les quatre flores de la France... Poinson-lès-Grancey.

Greuter, W. \& Rankin Rodríguez, R. (Eds.) (2018). Código Internacional de Nomenclatura para algas, hongos y plantas (Código de Shenzhen), 19ำ Congreso Internacional de Botánica Shenzhen, China (2017) - edición en español - Berlín: Fundación Herbarium Greuter.

Laínz, M. (1959). Aportaciones al conocimiento de la flora cántabro-astur. III. Collectanea Botanica (Barcelona), 5, 671-696.

Lambinon \& Verloove (2004). Agrostis $\times$ fouilladeana. Bulletin Société pour l'échange des plantes vasculaires de l'Europe occidentale et du bassin méditerranéen, 29, 105-109.

Paunero, E. (1948). Las especies españolas del género Agrostis. Anales del Jardín Botánico de Madrid, 7, 561-644.

Paunero, E. (1956). Las aveneas españolas I. Anales del Instituto Botánico A. J. Cavanilles, 13, 149-229.

Romero-García, A.T. (2011). Agrostis L. En: G. Blanca et al. (eds.), Flora Vascular de Andalucía Oriental (2a Edición corregida y aumentada), 331354. Universidades de Almería, Granada, Jaén y Málaga, Granada.

Romero-García, A.T., Blanca López, G. \& Morales Torres, C. (1988). Revisión del género Agrostis L. (Poaceae) en la Península Ibérica. Ruizia, 7, 47152.

Romero-Zarco, C. (1987). Agrostis L. En: B. Valdés, S. Talavera \& E.F. Galiano (eds.), Flora Vascular de Andalucía Occidental, 3, 336-341. Barcelona: Ketres Editores.

Romero-Zarco, C. (2015). Las Gramíneas de la Península Ibérica e Islas Baleares. Claves ilustradas para la determinación de los géneros y catálogo preliminar de las especies. Jaca: Jolube.

Sáez, L., López-Alvarado, J., Fraga, P., Berjano, R., Ortiz, M.A. \& Romero-Zarco, C. (2020a). Two New Species of Aira (Poaceae) from the Iberian Peninsula and the Balearic Islands. Systematic 
Botany, 45(1), 75-84.

Sáez, L., López-Alvarado, J., Fraga, P., Berjano, R., Ortiz, M.Á. \& Romero-Zarco, C. (2020b). Data from: Two New Species of Aira (Poaceae) from the Iberian Peninsula and the Balearic Islands. Dryad, Dataset,

https://doi.org/10.5061/dryad.69p8cz8x6

Sáez, L., Ortiz, M.Á. \& Romero Zarco, C. (2020c). Aira
L. En: J.A. Devesa et al. (eds.) Flora iberica XIX(I). Gramineae (partim), 473-489.

Tropicos.org. Missouri Botanical Garden. http://www.tropicos.org. Saint Louis, Missouri. Consultado el 1/02/2021.

Willkomm, H.M. (1861). Agrostis L. En: H.M. Willkomm \& J.M.C. Lange (Eds.) Prodromus Florae Hispanicae, 1, 51-56. Stuttgart. 